\title{
Synthesis and Single Crystal Structures of $N$-Substituted Benzamides and Their Chemoselective Selenation/Reduction Derivatives
}

\author{
Guoxiong Hua ${ }^{1}$, Cameron L. Carpenter-Warren ${ }^{1}$, David B. Cordes ${ }^{1} \mathbb{D}$, Alexandra M. Z. Slawin ${ }^{1,+(\mathbb{D})}$ \\ and J. Derek Woollins $1,2, * \mathbb{D}$
}

1 EaStCHEM School of Chemistry, University of St Andrews, St Andrews Fife KY16 9ST, UK; gh15@st-andrews.ac.uk (G.H.); clcw@st-andrews.ac.uk (C.L.C.-W.); dbc21@st-andrews.ac.uk (D.B.C.); amzs@st-andrews.ac.uk (A.M.Z.S.)

2 Department of Chemistry, Khalifa University, Abu Dhabi 127788, United Arab Emirates

* Correspondence: jdw3@st-andrews.ac.uk; Tel./Fax: +44-1334-463861

+ Dedication: The co-authors dedicate this paper to Prof. Slawin on the occasion of her 60th birthday.

Citation: Hua, G.; Carpenter-Warren, C.L.; Cordes, D.B.; Slawin, A.M.Z.; Woollins, J.D. Synthesis and Single Crystal Structures of $\mathrm{N}$-Substituted Benzamides and Their

Chemoselective

Selenation/Reduction Derivatives. Molecules 2021, 26, 2367.

https://doi.org/10.3390/

molecules 26082367

Academic Editors: Roberto Centore and Igor Djerdj

Received: 19 March 2021

Accepted: 15 April 2021

Published: 19 April 2021

Publisher's Note: MDPI stays neutral with regard to jurisdictional claims in published maps and institutional affiliations.

Copyright: (c) 2021 by the authors. Licensee MDPI, Basel, Switzerland. This article is an open access article distributed under the terms and conditions of the Creative Commons Attribution (CC BY) license (https:/ / creativecommons.org/licenses/by/ $4.0 /)$.

\begin{abstract}
A series of $\mathrm{N}$-aryl- $\mathrm{N}$-(2-oxo-2-arylethyl) benzamides and cinnamides has been prepared. The reaction of the benzamides with Woollins' reagent, a highly efficient chemoselective selenation/reduction reagent, gave the corresponding $N$-aryl- $N$-(arylenethyl) benzoselenoamides in good yields. Five representative single crystal $\mathrm{X}$-ray structures are discussed.
\end{abstract}

Keywords: N-Substituted Benzamides; Woollins' reagent; selenation reagent; reduction reagent; single crystal X-ray structures

\section{Introduction}

2,4-Bis (phenyl)-1,3-diselenadiphosphetane-2,4-diselenide (Woollins' reagent, WR) has played a role in synthetic chemistry in the past two decades [1-13]. It has been successfully applied as an efficient building unit to synthesize a series of eight-, nine-, and ten-membered selenophosphorus heterocycles with P-Se-Se-P linkage [14], as well as unique octaselenocyclododecane with four carbon atoms and eight selenium atoms in this twelve-membered cycle [15]. Another attractive application has been that it acts as a highly chemoselective reagent, e.g., the reduction of a wide range of 1,4-enediones and 1,4-ynediones in methanol led to saturated 1,4-diketones [16] and the selective reduction of the double bond of 2- $\alpha, \beta$-unsaturated thiazo- and selenazolidinones gave the corresponding saturated heterocycles [17]. Woollins' reagent has also been used as a reducting agent to transfer porpholactone into dihydroporpholactone or into adjacent-tetrahydroporpholactone [18].

Organoselenium compounds have received growing attention during the last decades due to their importance as useful precursors in synthetic chemistry $[19,20]$, as new synthetic materials [20], and their biological and medicinal significance [21]. To continue our interest in the chemistry of Woollins' reagent towards various organic substrates, we report an investigation on the use of WR as a selenation/reduction reagent for transferring $N$-aryl- $N$-(2-oxo-2-arylethyl) benzamides into the corresponding $N$-aryl- $N$-(arylenethyl) benzoselenoamides.

\section{Results and Discussion \\ 2.1. Synthesis and Characterization}

The synthesis of anilinoacetophenones 1-3, $N$-aryl- $N$-arylamidoacetophenones 4-6 and $N$-aryl- $N$-cinnamidoacetophenones 7-9 was carried out using a modified literature method [22]. The reaction of anilines and an equivalent of the appropriate bromoacetophenones in dry acetonitrile at room temperature gave anilinoacetophenones 1-3 in 81-87\% yields, respectively. Anilinoacetophenones $\mathbf{1}$ and $\mathbf{2}$ are new compounds, while $\mathbf{3}$ is a known 
compound, prepared previously by a similar method [23,24]; however, its single-crystal X-ray structure has not been reported previously. Acylation of anilinoacetophenones 1-3 with the appropriate acid chlorides in 1,2-dichloroethane at reflux led to the $\mathrm{N}$-aryl- $\mathrm{N}$ arylamidoacetophenones 4-6 and $\mathrm{N}$-aryl- $\mathrm{N}$-cinnamidoacetophenones $7-9$ in $76-91 \%$ yields, as shown in Scheme 1. All the new compounds were characterized by standard analytical and spectroscopic techniques. 1-9 show the anticipated $[\mathrm{M}]^{+}$or $[\mathrm{M}+\mathrm{H}]^{+}$peak in their mass spectra, satisfactory accurate mass measurements, and appropriate isotopic distributions; the ${ }^{1} \mathrm{H}$ NMR spectra display all the characteristic peaks of the phenyl backbones in compounds and the characteristic peaks of the $\mathrm{NH}$ group in compound $1-3$. The ${ }^{13} \mathrm{C}$ NMR spectra of compounds 1-9 display the characteristic signals of the $\mathrm{C}=\mathrm{O}$ groups.

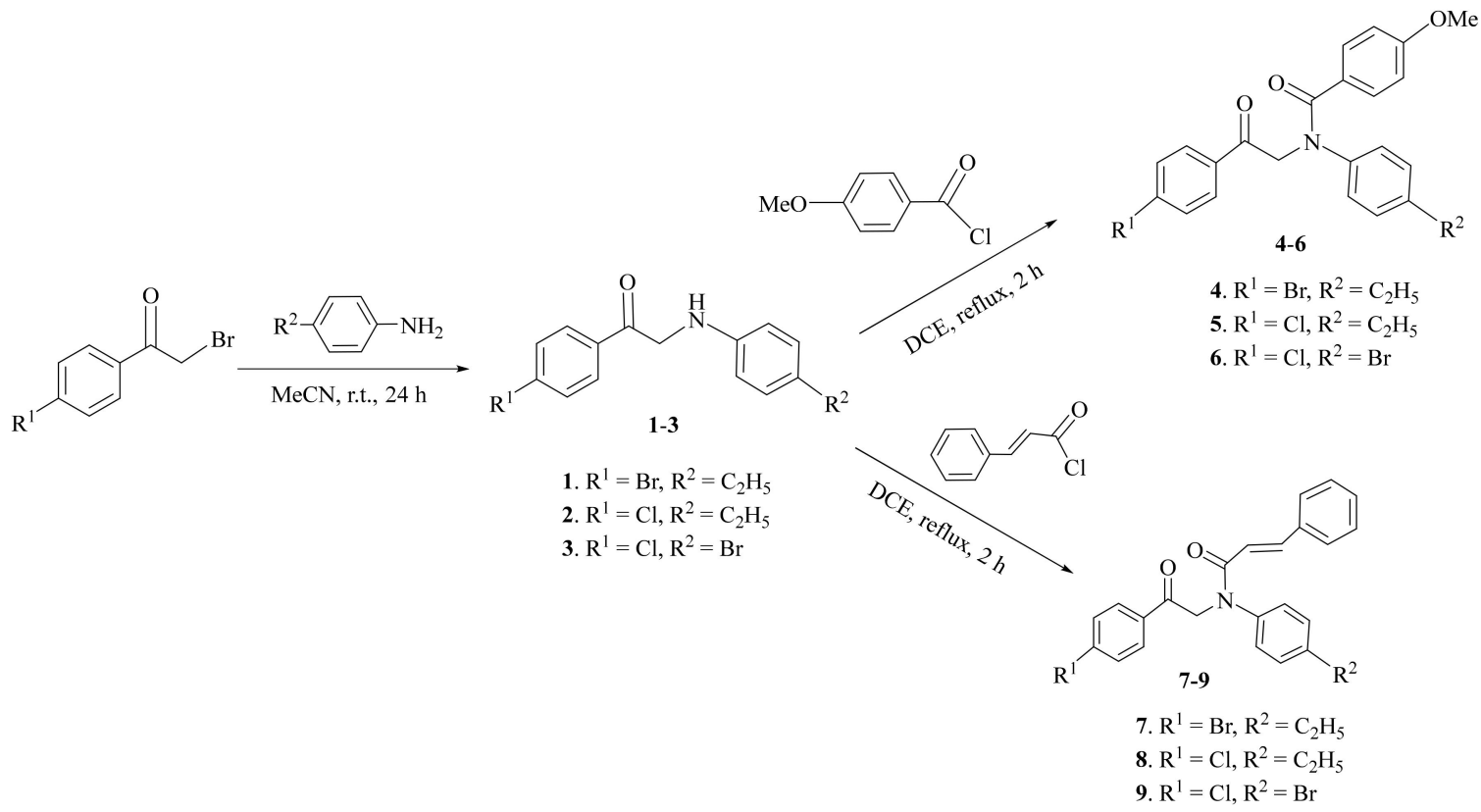

Scheme 1. Synthesis of anilinoacetophenones 1-3, N-aryl- $N$-arylamidoacetophenones $4-6$ and $N$-aryl- $N$-cinnamidoacetophenones 7-9.

Selenation of $\mathrm{N}$-aryl- $\mathrm{N}$-arylamidoacetophenones $\mathbf{4}-\mathbf{6}$ by WR gave rise to $\mathrm{N}$-aryl- $\mathrm{N}$ arylethylbenzoselenoamides $\mathbf{1 0 - 1 2}$ in $50 \%, 46 \%$ and $40 \%$ yields, respectively, rather than the expected 1,3-selenazole products (Scheme 2 ). One $\mathrm{C}=\mathrm{O}$ group has been converted to $\mathrm{C}=\mathrm{Se}$ and the other reduced to $\mathrm{CH}_{2}$ to give the final product $\mathrm{N}$-Aryl- $\mathrm{N}$-arylethylbenzoselenoamides 10-12. It is well known that WR is an efficient chemoselective reduction agent for diketones [16], $\alpha, \beta$-unsaturated thioazo and selenoazolidinones [6]. Based on the literature research and our findings, a possible mechanism for the selective reduction of $N$-substituted$\mathrm{N}$-phenylamidoacetophenones $\mathbf{4 - 6}$ is broadly similar to that of $\mathrm{NaSeH}$ and $\mathrm{LiSeH}$ as selective reducing agents of $\alpha, \beta$-unsaturated carbonyl compounds [13] and of PhSe-SePh as a reducing agent for electron deficient olefins [25], and it is probable that the reduction proceed through a Micheal reaction [26-28].

Reacting $\mathrm{N}$-aryl- $\mathrm{N}$-cinnamidoacetophenones 7-9 with WR under similar reaction conditions did not lead to a reaction, with the starting materials being recovered (Scheme 3). We speculate that the extra $\mathrm{C}=\mathrm{C}$ bond in $\mathrm{N}$-substituted- $\mathrm{N}$-phenylamidoacetophenones 7-9 which gives a conjugated structure, may be more stable and robust than $\mathrm{N}$-substituted-Nphenylamidoacetophenones 4-6 towards WR. 
<smiles>[R]c1ccc(C(=O)CN(C(=O)c2ccc(OC)cc2)c2ccc([R])cc2)cc1</smiles>

4. $\mathrm{R}^{1}=\mathrm{Br}, \mathrm{R}^{2}=\mathrm{C}_{2} \mathrm{H}_{5}$ $5 \mathrm{R}^{1}=\mathrm{Cl}, \mathrm{R}^{2}=\mathrm{C}_{2} \mathrm{H}_{5}$ 6. $\mathrm{R}^{1}=\mathrm{Cl}, \mathrm{R}^{2}=\mathrm{Br}$

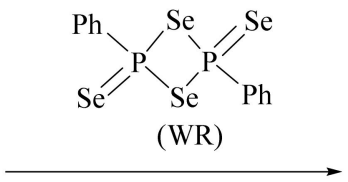

Toluene, reflux, $6 \mathrm{~h}$<smiles>[R]c1ccc(CCN(C(=S)c2ccc(OC)cc2)c2ccc([R])cc2)cc1</smiles>

10, $\mathrm{R}^{1}=\mathrm{Br}, \mathrm{R}^{2}=\mathrm{C}_{2} \mathrm{H}_{5}$ 11, $\mathrm{R}^{1}=\mathrm{Cl}, \mathrm{R}^{2}=\mathrm{C}_{2} \mathrm{H}_{5}$ 12, $\mathrm{R}^{1}=\mathrm{Cl}, \mathrm{R}^{2}=\mathrm{Br}$

Scheme 2. Selenation of $N$-aryl- $N$-phenylamidoacetophenones 4-6.
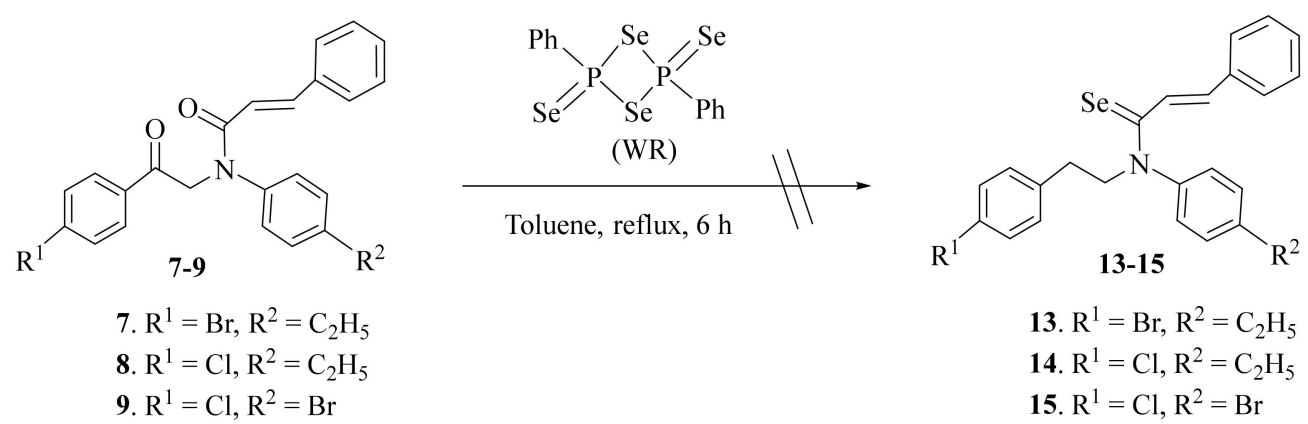

13. $\mathrm{R}^{1}=\mathrm{Br}, \mathrm{R}^{2}=\mathrm{C}_{2} \mathrm{H}_{5}$ 14. $\mathrm{R}^{1}=\mathrm{Cl}, \mathrm{R}^{2}=\mathrm{C}_{2} \mathrm{H}_{5}$ 15. $\mathrm{R}^{1}=\mathrm{Cl}, \mathrm{R}^{2}=\mathrm{Br}$

Scheme 3. Attempted selenation of $N$-aryl- $N$-cinnamidoacetophenones 7-9.

The new selenium derivatives $\mathbf{1 0 - 1 2}$ are quite stable both as solids and in solution, in air and moist atmospheres, and are soluble in common organic solvents. They show the anticipated molecular ion peaks $[\mathrm{M}+\mathrm{H}]^{+}$in their CI spectra and $[\mathrm{M}]^{+}$in their EI spectra, and satisfactory accurate mass measurements (EI). All the characteristic peaks of the phenyl backbones were found, and the characteristic peaks of the $\mathrm{NH}$ group disappeared in their ${ }^{1} \mathrm{H}$ NMR spectra. Their ${ }^{13} \mathrm{C}$ NMR spectra all show the normal signals for the $\mathrm{C}=$ Se groups $\left(\delta_{C}, 204.8-206.8 \mathrm{ppm}\right)$. Their ${ }^{77}$ Se NMR spectra exhibit singlet signals at $\delta_{\mathrm{Se}}=598.4,601.5$ and $601.4 \mathrm{ppm}$ for 10-12, respectively.

\subsection{Single Crystal Structure Analysis}

Single crystals of $\mathbf{3}, \mathbf{7}, \mathbf{1 0}-\mathbf{1 2}$ suitable for X-ray crystallographic analysis were grown by slow evaporation of dichloromethane solutions of the compound in air at room temperature. Selected crystallographic data are given in Table 1 and the resulting molecular structures are illustrated in Figures 1 and 2.

The molecular structure of anilinoacetophenone 3 (Figure 1) shows a planar arrangement, with a mean deviation of non-hydrogen atoms from the plane of $0.047 \AA$. Adjacent molecules of 3 interact to form hydrogen-bonded dimers via a pair of $\mathrm{NH} \cdots \mathrm{O}$ hydrogen

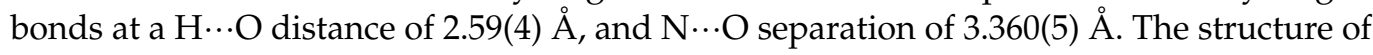
the $\mathrm{N}$-aryl- $\mathrm{N}$-cinnamidoacetophenone $\mathbf{7}$ shows the compound in a twisted-T conformation (Figure 1). As expected, the acetophenone group retains its planarity (mean deviation of non-hydrogen atoms from the plane of $0.004 \AA$ ). Meanwhile, the other two phenyl ring planes are twisted out of the acetophenone plane, with angles between planes of 71.36 and $50.74^{\circ}$ for $\mathrm{C}(10)-\mathrm{C}(15)$ and $\mathrm{C}(21)-\mathrm{C}(26)$, respectively. 
Table 1. Details of the X-ray data collections and refinements for compounds 3, 7, 10-12.

\begin{tabular}{|c|c|c|c|c|c|}
\hline Compound & 3 & 7 & 10 & 11 & 12 \\
\hline Formula & $\mathrm{C}_{14} \mathrm{H}_{11} \mathrm{BrClNO}$ & $\mathrm{C}_{25} \mathrm{H}_{22} \mathrm{ClNO}_{2}$ & $\mathrm{C}_{24} \mathrm{H}_{24} \mathrm{BrNOSe}$ & $\mathrm{C}_{24} \mathrm{H}_{24} \mathrm{ClNOSe}$ & $\mathrm{C}_{22} \mathrm{H}_{19} \mathrm{BrClNOSe}$ \\
\hline$M$ & 324.60 & 403.91 & 501.32 & 456.87 & 507.72 \\
\hline Temperature/K & 93 & 93 & 173 & 173 & 173 \\
\hline Crystal system & triclinic & monoclinic & triclinic & triclinic & triclinic \\
\hline Space group & $P \overline{1}$ & $P 2_{1} / c$ & $P \overline{1}$ & $P \overline{1}$ & $P \overline{1}$ \\
\hline $\mathrm{a} / \AA$ & $5.718(3)$ & $6.00023(18)$ & $9.2004(17)$ & $9.1375(12)$ & $9.98830(10)$ \\
\hline $\mathrm{b} / \AA$ & $7.296(4)$ & $22.8404(6)$ & $10.541(3)$ & $10.6864(14)$ & $10.0160(2)$ \\
\hline$c / \AA$ & $15.461(8)$ & $14.7270(4)$ & $11.146(3)$ & $11.1485(15)$ & $12.3778(18)$ \\
\hline$\alpha$ & $95.472(13)$ & & $101.842(4)$ & $103.062(4)$ & $99.855(14)$ \\
\hline$\beta$ & $90.3200(10)$ & $93.111(3)$ & $94.351(6)$ & $95.439(3)$ & $110.822(8)$ \\
\hline$\gamma$ & $906.126(12)$ & & $90.478(6)$ & $91.412(4)$ & $108.988(12)$ \\
\hline $\mathrm{U} / \mathrm{A}^{3}$ & $638.3(6)$ & $2015.33(10)$ & $1054.6(5)$ & $1054.5(2)$ & $1035.2(2)$ \\
\hline Z & 2 & 4 & 2 & 2 & 2 \\
\hline$\mu / \mathrm{mm}^{-1}$ & 3.424 & 0.211 & 3.695 & 1.922 & 3.890 \\
\hline $\begin{array}{l}\text { Reflections } \\
\text { collected }\end{array}$ & 8491 & 34,715 & 14,106 & 21,665 & 21,452 \\
\hline $\begin{array}{l}\text { Independent } \\
\text { reflections }\end{array}$ & 2296 & 4515 & 3785 & 3832 & 3776 \\
\hline$R_{\text {int }}$ & 0.0769 & 0.0669 & 0.0448 & 0.0283 & 0.0314 \\
\hline$R_{1}[I>2 \sigma(I)]$ & 0.0529 & 0.0341 & 0.0240 & 0.0304 & 0.0207 \\
\hline$w R_{2}$ & 0.1479 & 0.0883 & 0.0768 & 0.0973 & 0.0569 \\
\hline
\end{tabular}
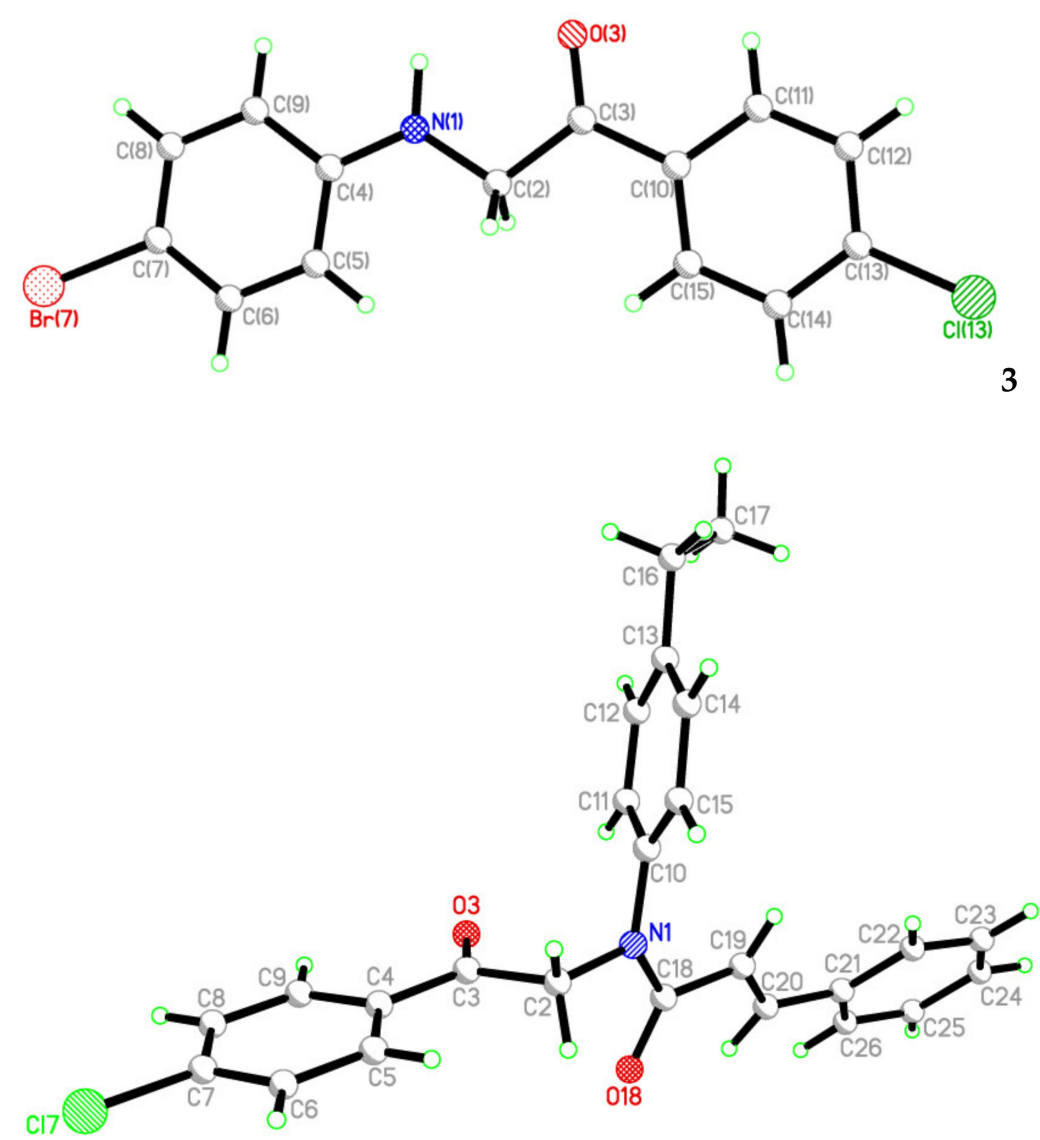

7

Figure 1. Single crystal X-ray structures of anilinoacetophenone 3 and $N$-aryl-Ncinnamidoacetophenone 7. 

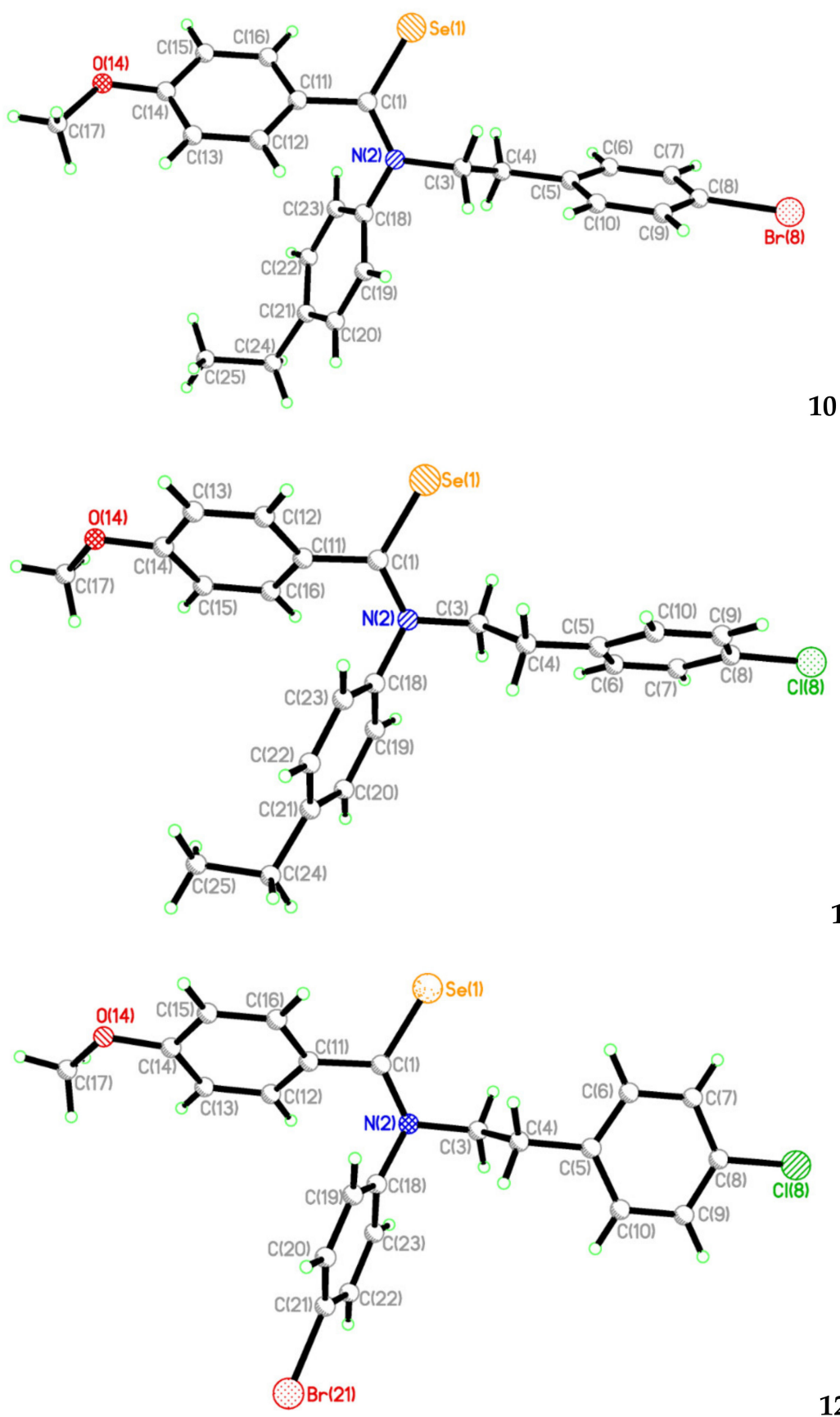

Figure 2. Single crystal X-ray structures of selenoamides 10, 11 and 12.

The X-ray structures of 10, 11 and 12 are depicted in Figure 2, each displaying similar twisted-T conformations. The three aryl rings $[\mathrm{C}(5)-\mathrm{C}(10)$ (ring 1), $\mathrm{C}(11)-\mathrm{C}(16)$ (ring 2) and $\mathrm{C}(18)-\mathrm{C}(23)$ (ring 3) in all three compounds] are all twisted with respect to each other, the angles between ring planes being 28.92, 69.02 and $62.00^{\circ}$ in 10, 18.92, 62.23 and $64.51^{\circ}$ in 11 and $55.76,61.91$ and $68.23^{\circ}$ in $\mathbf{1 2}$ for rings 1 and 2, 1 and 3 , and 2 and 3 , respectively. This pattern of ring twists puts all the rings out of the plane of the selenoamide, except for ring 1 in compound 12, which is near parallel to the selenoamide, inclined at $9.24^{\circ}$. The $\mathrm{C}=\mathrm{Se}$ double bond lengths [1.832(3) $\AA$ in 10, 1.833(2) $\AA$ in 11 and 1.8264(19) $\AA$ in 12] are very similar, falling at the middle of the range of $\mathrm{C}=$ Se bonds in known selenoamides [1.81(5) to 1.856(4) ̊]. [29] The conformations of 10-12 are all very similar, that between $\mathbf{1 0}$ and 11, being almost identical. The difference between this conformation and that adopted by 12 is in the orientation of ring 1 , which differs by $\sim 67^{\circ}$ between the two conformations. 


\section{Materials and Methods}

\subsection{General}

Unless otherwise stated, all reactions were carried out under an oxygen-free nitrogen atmosphere using pre-dried solvents and standard Schlenk techniques, subsequent chromatographic and work up procedures were performed in air. ${ }^{1} \mathrm{H}(400.1 \mathrm{MHz}),{ }^{13} \mathrm{C}$ $(100.6 \mathrm{MHz})$ and ${ }^{77} \mathrm{Se}-\left\{{ }^{1} \mathrm{H}\right\}\left(51.5 \mathrm{MHz}\right.$, referenced to external $\left.\mathrm{Me}_{2} \mathrm{Se}\right) \mathrm{NMR}$ spectra were recorded at $25{ }^{\circ} \mathrm{C}$ (NMR Jeol GSX270). IR spectra were recorded as $\mathrm{KBr}$ pellets in the range of $4000-250 \mathrm{~cm}^{-1}$ on a Perkin-Elmer 2000 FTIR/Raman spectrometer Mass spectrometry was performed by the EPSRC National Mass Spectrometry Service Centre, Swansea.

\section{Crystallography}

X-ray diffraction data for compounds 3, 7 and 10-12 were collected at either $93 \mathrm{~K}$ or $173 \mathrm{~K}$ using a Rigaku FR-X Ultrahigh Brilliance Microfocus RA generator/confocal optics with XtaLAB P200 diffractometer [Mo K $\alpha$ radiation $(\lambda=0.71075 \AA)$ ]. Intensity data were collected using $\omega$ steps accumulating area detector images spanning at least a hemisphere of reciprocal space. Data for all compounds were collected using CrystalClear 2.1 [30] and processed (including correction for Lorentz, polarization and absorption) using either CrystalClear [30] or CrysAlisPro 1.171.38.43. [31] Structures were solved by dual-space (SHELXT-2014 / 4 [32]) or Patterson (PATTY [33]) methods and refined by fullmatrix least-squares against $\mathrm{F}^{2}$ (SHELXL-2018/3 [34]). Non-hydrogen atoms were refined anisotropically, and hydrogen atoms were refined using a riding model, except for the amine hydrogen in 3 which was located from the difference Fourier map and refined isotropically subject to a distance restraint. All calculations were performed using the CrystalStructure 4.3 interface [35]. Selected crystallographic data are presented in Table 1. Deposition numbers 2071413-2071417 contains the supplementary crystallographic data for this paper. These data are provided free of charge by the joint Cambridge Crystallographic Data Centre and Fachinformationszentrum Karlsruhe Access Structures service www.ccdc. cam.ac.uk/structures.

\subsection{Synthesis}

\subsubsection{General Procedure for Synthesis of Compounds 1-3}

The appropriate aniline $(20 \mathrm{mmol})$ and the phenacyl bromide $(10 \mathrm{mmol})$ were combined in $\mathrm{MeCN}(40 \mathrm{~mL})$ and allowed to stir at room temperature for $24 \mathrm{~h}$. The amine salt was filtered off and the filtrate was concentrated under vacuum. The residue was dissolved in EtOAc $(40 \mathrm{~mL})$ and washed sequentially with $\mathrm{H}_{2} \mathrm{O}(50 \mathrm{~mL}), 5 \%$ citric acid $(50 \mathrm{~mL})$ and brine $(25 \mathrm{~mL})$. The organic layer was dried over $\mathrm{Na}_{2} \mathrm{SO}_{4}$, filtered through a pad of silica gel and the solvent was evaporated to give the product aminoacetophenones 1-3 in good yield.

1-(4-Bromophenyl)-2-((4-ethylphenyl)amino)ethan-1-one (1). Brown solid (83\% yield). M.p. $151-153{ }^{\circ} \mathrm{C}$. Selected IR (KBr, cm $\left.{ }^{-1}\right), 1679(\mathrm{vs}), 1617(\mathrm{~s}), 1583(\mathrm{~s}), 1522(\mathrm{vs}), 1585(\mathrm{~m})$, 1388(m), 1351(m), 1311(m), 1216(m), 1178(m), 1140(m), 1068(s), 992(vs), 812(vs), 576(m), $501(\mathrm{~m}) .{ }^{1} \mathrm{H} \mathrm{NMR}\left(\mathrm{CDCl}_{3}, \delta\right), 7.80(\mathrm{~d}, J(\mathrm{H}, \mathrm{H})=8.6 \mathrm{~Hz}, 2 \mathrm{H}), 7.69(\mathrm{~d}, J(\mathrm{H}, \mathrm{H})=8.4 \mathrm{~Hz}, 2 \mathrm{H})$, $7.32(\mathrm{~s}, 1 \mathrm{H}), 7.09(\mathrm{~d}, J(\mathrm{H}, \mathrm{H})=8.6 \mathrm{~Hz}, 2 \mathrm{H}), 6.68(\mathrm{~d}, J(\mathrm{H}, \mathrm{H})=8.6 \mathrm{~Hz}, 2 \mathrm{H}), 4.60(\mathrm{~s}, 2 \mathrm{H}), 2.58(\mathrm{~d}$, $J(\mathrm{H}, \mathrm{H})=7.6 \mathrm{~Hz}, 2 \mathrm{H}), 1.22(\mathrm{~d}, J(\mathrm{H}, \mathrm{H})=7.6 \mathrm{~Hz}, 3 \mathrm{H}) \mathrm{ppm} .{ }^{13} \mathrm{C} \mathrm{NMR}\left(\mathrm{CDCl}_{3}, \delta\right), 194.4,145.0$, $133.9,133.7,132.2,129.3,129.0,128.7,113.2,50.7,28.0,16.0$ ppm. Accurate mass measurement $\left[\mathrm{ESI}^{+}, m / z\right]$ : found $318.0482[\mathrm{M}+\mathrm{H}]^{+}$, calculated mass for $\mathrm{C}_{16} \mathrm{H}_{16} \mathrm{BrNOH}$ : 318.0486 .

1-(4-Chlorophenyl)-2-((4-ethylphenyl)amino)ethan-1-one (2). Dark yellow solid (81\% yield). M.p. $148-149{ }^{\circ} \mathrm{C}$. Selected IR (KBr, cm $\left.{ }^{-1}\right), 1678(\mathrm{vs}), 1618(\mathrm{~m}), 1598(\mathrm{~s}), 1522(\mathrm{~s})$, 1489(m), 1440(m), 1395(m), 1352(s), 1312(m), 1218(s), 1090(s), 993(s), 815(vs), 577(m), $529(\mathrm{~m}) .{ }^{1} \mathrm{H} \mathrm{NMR}\left(\mathrm{CDCl}_{3}, \delta\right), 7.99(\mathrm{~d}, J(\mathrm{H}, \mathrm{H})=8.6 \mathrm{~Hz}, 2 \mathrm{H}), 7.52(\mathrm{~d}, J(\mathrm{H}, \mathrm{H})=8.6 \mathrm{~Hz}$, $2 \mathrm{H}), 7.32(\mathrm{~s}, 1 \mathrm{H}), 7.09(\mathrm{~d}, J(\mathrm{H}, \mathrm{H})=8.4 \mathrm{~Hz}, 2 \mathrm{H}), 6.68(\mathrm{~d}, J(\mathrm{H}, \mathrm{H})=8.4 \mathrm{~Hz}, 2 \mathrm{H}), 4.41(\mathrm{~s}$,

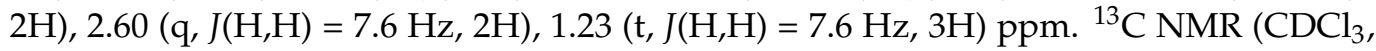
ઈ), 194.2, 145.0, 140.3, 133.9, 133.3, 129.3, 129.2, 129.1, 128.7, 113.2, 50.8, 28.0, 16.0 ppm. Accurate mass measurement $\left[\mathrm{ESI}^{+}, \mathrm{m} / z\right]$ : found $274.0992[\mathrm{M}+\mathrm{H}]^{+}$, calculated mass for $\mathrm{C}_{16} \mathrm{H}_{16} \mathrm{ClNOH}: 274.0994$. 
2-((4-Bromophenyl)amino)-1-(4-chlorophenyl)ethan-1-one (3). Greenish yellow solid (87\% yield). M.p. $165-166^{\circ}$ C. Selected IR (KBr, cm $\left.{ }^{-1}\right), 1678(v s), 1595(\mathrm{~s}), 1510(\mathrm{~s}), 1491(\mathrm{~s})$, 1400(m), 1357(s), 1256(m), 1218(m), 1094(s), 991(s), 814(s), 797(m), 574(m), 499(m). ${ }^{1} \mathrm{H}$ $\operatorname{NMR}\left(\mathrm{CDCl}_{3}, \delta\right), 7.98(\mathrm{~d}, J(\mathrm{H}, \mathrm{H})=8.6 \mathrm{~Hz}, 2 \mathrm{H}), 7.52(\mathrm{~d}, J(\mathrm{H}, \mathrm{H})=8.6 \mathrm{~Hz}, 2 \mathrm{H}), 7.32(\mathrm{~d}$, $J(\mathrm{H}, \mathrm{H})=8.4 \mathrm{~Hz}, 2 \mathrm{H}), 7.28(\mathrm{~s}, 1 \mathrm{H}), 6.60(\mathrm{~d}, J(\mathrm{H}, \mathrm{H})=8.4 \mathrm{~Hz}, 2 \mathrm{H}), 4.56(\mathrm{~s}, 2 \mathrm{H}) \mathrm{ppm} .{ }^{13} \mathrm{C}$ $\mathrm{NMR}\left(\mathrm{CDCl}_{3}, \delta\right), 194.2,145.0,140.3,133.9,133.3,129.3,129.2,129.2,128.7,113.2,50.8,28.0$, 16.0 ppm. Accurate mass measurement $\left[\mathrm{ESI}^{+}, \mathrm{m} / z\right]$ : found $323.9789[\mathrm{M}+\mathrm{H}]^{+}$, calculated mass for $\mathrm{C}_{14} \mathrm{H}_{11} \mathrm{BrClNOH}$ : 323.9791.

\subsubsection{General Procedure for Synthesis of Compounds 4-9}

The appropriate aminoacetophenone $(5.0 \mathrm{mmol})$ was dissolved in dichloroethane $(25 \mathrm{~mL})$ and refluxed for $2 \mathrm{~h}$ with the appropriate acid chloride (5.0 equiv). Volatiles were evaporated in vacuo, and the residue was recrystallized from ethyl acetate to give the expected products $\mathbf{4 - 9 .}$

N-(2-(4-Bromophenyl)-2-oxoethyl)-N-(4-ethylphenyl)-4-methoxybenzamide (4). Yellow solid (91\% yield). M.p. 127-129 ${ }^{\circ} \mathrm{C}$. Selected IR (KBr, $\left.\mathrm{cm}^{-1}\right), 1678(\mathrm{vs}), 1617(\mathrm{~m}), 1590(\mathrm{~m})$, 1521(s), 1352(m), 1306(m), 1262(m), 1219(m), 1091(m), 994(s), 844(m), 815(s), 772(m), 696(m), 613(m), 546(m), 503(m). ${ }^{1} \mathrm{H} \mathrm{NMR}\left(\mathrm{CDCl}_{3}, \delta\right), 8.09(\mathrm{~d}, J(\mathrm{H}, \mathrm{H})=8.9 \mathrm{~Hz}, 2 \mathrm{H}), 7.90(\mathrm{~d}$, $J(\mathrm{H}, \mathrm{H})=8.7 \mathrm{~Hz}, 2 \mathrm{H}), 7.68(\mathrm{~d}, J(\mathrm{H}, \mathrm{H})=8.6 \mathrm{~Hz}, 2 \mathrm{H}), 7.09(\mathrm{~d}, J(\mathrm{H}, \mathrm{H})=8.6 \mathrm{~Hz}, 2 \mathrm{H}), 6.97$ $(\mathrm{d}, J(\mathrm{H}, \mathrm{H})=8.9 \mathrm{~Hz}, 2 \mathrm{H}), 6.68(\mathrm{~d}, J(\mathrm{H}, \mathrm{H})=8.5 \mathrm{~Hz}, 2 \mathrm{H}), 4.59(\mathrm{~s}, 2 \mathrm{H}), 3.91(\mathrm{~s}, 3 \mathrm{H}), 2.59(\mathrm{q}$, $J(\mathrm{H}, \mathrm{H})=7.6 \mathrm{~Hz}, 2 \mathrm{H}), 1.22(\mathrm{t}, J(\mathrm{H}, \mathrm{H})=7.6 \mathrm{~Hz}, 3 \mathrm{H}) \mathrm{ppm} .{ }^{13} \mathrm{C} \mathrm{NMR}\left(\mathrm{CDCl}_{3}, \delta\right), 194.5,171.3$, 164.2, 145.0, 133.9, 133.7, 132.4, 133.2, 132.0, 129.3, 129.0, 128.7, 121.7, 113.8, 113.3, 55.5, 50.8, 28.0, 16.0 ppm. Accurate mass measurement $\left[\mathrm{CI}^{+}, \mathrm{m} / z\right]$ : found $452.0859[\mathrm{M}+\mathrm{H}]^{+}$, calculated mass for $\mathrm{C}_{24} \mathrm{H}_{22} \mathrm{BrNO}_{3} \mathrm{H}: 452.0861$.

$N$-(2-(4-Chlorophenyl)-2-oxoethyl)- $N$-(4-ethylphenyl)-4-methoxybenzamide (5). Bright yellow solid (81\% yield). M.p. $126-128{ }^{\circ} \mathrm{C}$. Selected IR (KBr, cm $\left.{ }^{-1}\right), 1678(\mathrm{vs}), 1617(\mathrm{~s})$, 1590(s), 1512(vs), 1396(m), 1352(m), 1307(m), 1262(m), 1218(m), 1179(m), 1090(s), 994(s), 815(s), 772(m), 613(m), 578(m), 546(m), 503(m). ${ }^{1} \mathrm{H} \mathrm{NMR}\left(\mathrm{CDCl}_{3}, \delta\right), 8.10(\mathrm{~d}, J(\mathrm{H}, \mathrm{H})=8.9 \mathrm{~Hz}$, $2 \mathrm{H}), 7.99(\mathrm{~d}, J(\mathrm{H}, \mathrm{H})=8.6 \mathrm{~Hz}, 2 \mathrm{H}), 7.51(\mathrm{~d}, J(\mathrm{H}, \mathrm{H})=8.6 \mathrm{~Hz}, 2 \mathrm{H}), 7.09(\mathrm{~d}, J(\mathrm{H}, \mathrm{H})=8.4 \mathrm{~Hz}$, $2 \mathrm{H}), 6.97(\mathrm{~d}, J(\mathrm{H}, \mathrm{H})=8.9 \mathrm{~Hz}, 2 \mathrm{H}), 6.68(\mathrm{~d}, J(\mathrm{H}, \mathrm{H})=8.4 \mathrm{~Hz}, 2 \mathrm{H}), 4.61(\mathrm{~s}, 2 \mathrm{H}), 3.91(\mathrm{~s}, 3 \mathrm{H})$, $2.59(\mathrm{q}, J(\mathrm{H}, \mathrm{H})=7.6 \mathrm{~Hz}, 2 \mathrm{H}), 1.23(\mathrm{t}, J(\mathrm{H}, \mathrm{H})=7.6 \mathrm{~Hz}, 3 \mathrm{H}) \mathrm{ppm} .{ }^{13} \mathrm{C} \mathrm{NMR}\left(\mathrm{CDCl}_{3}, \delta\right), 194.2$, 171.5, 164.0, 145.0, 140.3, 133.9, 133.3, 132.4, 129.3, 129.2, 129.0, 128.8, 121.7, 113.7, 113.2, 55.5, 50.8, 28.0, 16.0 ppm. Accurate mass measurement $\left[\mathrm{CI}^{+}, \mathrm{m} / z\right]$ : found $408.1366[\mathrm{M}+\mathrm{H}]^{+}$, calculated mass for $\mathrm{C}_{24} \mathrm{H}_{22} \mathrm{ClNO}_{3} \mathrm{H}: 408.1367$.

$N$-(4-Bromophenyl)-N-(2-(4-chlorophenyl)-2-oxoethyl)-4-methoxybenzamide (6). Gray solid (83\% yield). M.p. 150-152 ${ }^{\circ} \mathrm{C}$. Selected IR (KBr, $\left.\mathrm{cm}^{-1}\right), 1680(\mathrm{~s}), 1601(\mathrm{~s}), 1574(\mathrm{~m})$, 1513(m), 1487(m), 1427(s), 1301(s), 1260(s), 1166(s), 1025(m), 926(m), 844(s), 816(m), 772(s), 696(m), 613(s), 547(s), 503(m), 484(m). ${ }^{1} \mathrm{H}$ NMR $\left(\mathrm{CDCl}_{3}, \delta\right), 8.09(\mathrm{~d}, J(\mathrm{H}, \mathrm{H})=8.9 \mathrm{~Hz}, 2 \mathrm{H})$, $7.98(\mathrm{~d}, J(\mathrm{H}, \mathrm{H})=8.6 \mathrm{~Hz}, 2 \mathrm{H}), 7.52(\mathrm{~d}, J(\mathrm{H}, \mathrm{H})=8.6 \mathrm{~Hz}, 2 \mathrm{H}), 7.32(\mathrm{~d}, J(\mathrm{H}, \mathrm{H})=8.9 \mathrm{~Hz}, 2 \mathrm{H})$, $6.97(\mathrm{~d}, J(\mathrm{H}, \mathrm{H})=8.8 \mathrm{~Hz}, 2 \mathrm{H}), 6.61(\mathrm{~d}, J(\mathrm{H}, \mathrm{H})=8.8 \mathrm{~Hz}, 2 \mathrm{H}), 4.57(\mathrm{~s}, 2 \mathrm{H}), 3.91(\mathrm{~s}, 3 \mathrm{H}) \mathrm{ppm}$. ${ }^{13} \mathrm{C} \mathrm{NMR}\left(\mathrm{CDCl}_{3}, \delta\right), 193.5,171.3,164.0,145.8,140.6,133.0,132.4,132.1,129.3,129.2,121.6$, $114.7,113.8,110.8,109.7,55.5,50.2 \mathrm{ppm}$. Accurate mass measurement $\left[\mathrm{CI}^{+}, \mathrm{m} / z\right]$ : found $458.0157[\mathrm{M}+\mathrm{H}]^{+}$, calculated mass for $\mathrm{C}_{22} \mathrm{H}_{17} \mathrm{BrClNO}_{3} \mathrm{H}: 458.0159$.

$\mathrm{N}$-(2-(4-Bromophenyl)-2-oxoethyl)- $N$-(4-ethylphenyl)cinnamamide (7). Yellow solid (79\% yield). M.p. 126-127 ${ }^{\circ}$ C. Selected IR (KBr, cm $\left.{ }^{-1}\right), 1697(\mathrm{~s}), 1656(\mathrm{vs}), 1620(\mathrm{~s}), 1584(\mathrm{~s}), 1510(\mathrm{~s})$, 1401(m), 1376(s), 1327(m), 1209(s), 1069(s), 1005(s), 982(s), 840(m), 812(s), 703(s), 698(s), 569(m), 549(s). ${ }^{1} \mathrm{H} \mathrm{NMR}\left(\mathrm{CDCl}_{3}, \delta\right), 7.87(\mathrm{~d}, J(\mathrm{H}, \mathrm{H})=7.6 \mathrm{~Hz}, 2 \mathrm{H}), 7.73(\mathrm{~d}, J(\mathrm{H}, \mathrm{H})=15.6 \mathrm{~Hz}, 1 \mathrm{H})$, $7.63(\mathrm{~d}, J(\mathrm{H}, \mathrm{H})=7.8 \mathrm{~Hz}, 2 \mathrm{H}), 7.38-7.26(\mathrm{~m}, 8 \mathrm{H}), 6.49(\mathrm{~d}, J(\mathrm{H}, \mathrm{H})=15.6 \mathrm{~Hz}, 1 \mathrm{H}), 5.18(\mathrm{~s}$, 2H), $2.72(\mathrm{q}, J(\mathrm{H}, \mathrm{H})=7.6 \mathrm{~Hz}, 2 \mathrm{H}), 1.29(\mathrm{t}, J(\mathrm{H}, \mathrm{H})=7.6 \mathrm{~Hz}, 3 \mathrm{H}) \mathrm{ppm} .{ }^{13} \mathrm{C} \mathrm{NMR}\left(\mathrm{CDCl}_{3}, \delta\right)$, $192.9,166.4,144.3,142.7,140.0,135.1,134.1,132.1,129.9,129.6,129.5,129.1,128.7,128.1$, $128.0,118.0,56.5,28.5,15.4 \mathrm{ppm}$. Accurate mass measurement $\left[\mathrm{CI}^{+}, m / z\right]$ : found 448.0916 $[\mathrm{M}+\mathrm{H}]^{+}$, calculated mass for $\mathrm{C}_{25} \mathrm{H}_{22} \mathrm{BrNO}_{2} \mathrm{H}: 448.0912$.

N-(2-(4-Chlorophenyl)-2-oxoethyl)-N-(4-ethylphenyl)cinnamamide (8). Dark yellow solid (80\% yield). M.p. $155-157^{\circ}$ C. Selected IR (KBr, cm $\left.{ }^{-1}\right)$, 1697(s), 1653(s), 1617(s), 1587(s), 
1510(s), 1402(m), 1380(s), 1329(m), 1214(s), 1089(s), 1000(m), 981(s), 819(s), 766(m), 703(m), 548(m), 525(m). ${ }^{1} \mathrm{H}$ NMR $\left(\mathrm{CDCl}_{3}, \delta\right), 7.92(\mathrm{~d}, J(\mathrm{H}, \mathrm{H})=7.6 \mathrm{~Hz}, 2 \mathrm{H}), 7.70(\mathrm{~d}, J(\mathrm{H}, \mathrm{H})=15.1 \mathrm{~Hz}$, $1 \mathrm{H}), 7.43(\mathrm{~d}, J(\mathrm{H}, \mathrm{H})=7.8 \mathrm{~Hz}, 2 \mathrm{H}), 7.28-7.21(\mathrm{~m}, 8 \mathrm{H}), 6.45(\mathrm{~d}, J(\mathrm{H}, \mathrm{H})=16.1 \mathrm{~Hz}, 1 \mathrm{H}), 5.16$ $(\mathrm{s}, 2 \mathrm{H}), 2.68(\mathrm{q}, J(\mathrm{H}, \mathrm{H})=7.6 \mathrm{~Hz}, 2 \mathrm{H}), 1.25(\mathrm{t}, J(\mathrm{H}, \mathrm{H})=7.6 \mathrm{~Hz}, 3 \mathrm{H}) \mathrm{ppm} .{ }^{13} \mathrm{C} \mathrm{NMR}\left(\mathrm{CDCl}_{3}\right.$,

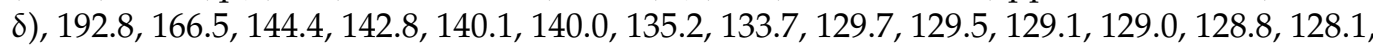
$128.0,118.0,56.6,28.6,15.5 \mathrm{ppm}$. Accurate mass measurement $\left[\mathrm{CI}^{+}, \mathrm{m} / z\right]$ : found 404.1408 $[\mathrm{M}+\mathrm{H}]^{+}$, calculated mass for $\mathrm{C}_{25} \mathrm{H}_{22} \mathrm{ClNO}_{2} \mathrm{H}: 404.1412$.

N-(4-Bromophenyl)-N-(2-(4-chlorophenyl)-2-oxoethyl)cinnamamide (9). Off-white solid (76\% yield). M.p. $174-176^{\circ} \mathrm{C}$. Selected IR (KBr, $\left.\mathrm{cm}^{-1}\right), 1696(\mathrm{~s}), 1657(\mathrm{~s}), 1617(\mathrm{~s})$, 1587(m), 1486(vs), 1408(m), 1373(s), 1207(s), 1092(m), 1070(m), 1013(s), 816(s), 763(m), 696(m), 484(s). ${ }^{1} \mathrm{H}$ NMR $\left(\mathrm{CDCl}_{3}, \delta\right), 7.95(\mathrm{~d}, J(\mathrm{H}, \mathrm{H})=8.6 \mathrm{~Hz}, 2 \mathrm{H}), 7.74(\mathrm{~d}, J(\mathrm{H}, \mathrm{H})=15.5 \mathrm{~Hz}$, $1 \mathrm{H}), 7.58(\mathrm{~d}, J(\mathrm{H}, \mathrm{H})=8.6 \mathrm{~Hz}, 2 \mathrm{H}), 7.48(\mathrm{~d}, J(\mathrm{H}, \mathrm{H})=8.6 \mathrm{~Hz}, 2 \mathrm{H}), 7.39-7.32(\mathrm{~m}, 6 \mathrm{H}), 6.46(\mathrm{~d}$, $J(\mathrm{H}, \mathrm{H})=15.5 \mathrm{~Hz}, 2 \mathrm{H}), 5.18(\mathrm{~s}, 2 \mathrm{H}) \mathrm{ppm} .{ }^{13} \mathrm{C} \mathrm{NMR}\left(\mathrm{CDCl}_{3}, \delta\right), 192.4,166.1,143.5,141.5$, $140.2,134.8,133.4,132.9,130.0,129.9,129.5,129.2,128.8,128.1,122.0,117.3,56.3$ ppm. Accurate mass measurement $\left[\mathrm{CI}^{+}, \mathrm{m} / z\right]$ : found $456.0184[\mathrm{M}+\mathrm{H}]^{+}$, calculated mass for $\mathrm{C}_{23} \mathrm{H}_{17} \mathrm{BrClNO}_{2} \mathrm{H}: 456.0189$.

\subsubsection{General Procedure for Synthesis of Compounds 10-12}

A mixture of the appropriate benzamide or cinnamide with an equivalent of $\mathbf{W R}$ in dry toluene was refluxed for $6 \mathrm{~h}$. Following cooling to room temperature and filtration to remove unreacted solid, the filtrate was evaporated in vacuo, the residue was dissolved in $2 \mathrm{~mL}$ of dichloromethane and purified by silica gel column chromatography (1:1 hexane/dichloromethane as eluant) to give the products 10-12. Cinnamides 7-9 did not show any reaction with $\mathbf{W R}$, returning the starting materials.

$\mathrm{N}$-(4-Bromophenethyl)-N-(4-ethylphenyl)-4-methoxybenzoselenoamide (10). Greyish yellow paste $(0.25 \mathrm{~g}, 50 \%)$. Selected IR (KBr, $\left.\mathrm{cm}^{-1}\right), 1601(\mathrm{~s}), 1508(\mathrm{~s}), 1488(\mathrm{~m}), 1448(\mathrm{~s}), 1399(\mathrm{~s})$, 1300(m), 1250(vs), 1170(s), 1073(m), 1029(m), 830(m), 806(m). ${ }^{1} \mathrm{H}$ NMR $\left(\mathrm{CD}_{2} \mathrm{Cl}_{2}, \delta\right), 8.03(\mathrm{~d}$, $J(\mathrm{H}, \mathrm{H})=8.0 \mathrm{~Hz}, 1 \mathrm{H}), 7.45(\mathrm{~d}, J(\mathrm{H}, \mathrm{H})=8.4 \mathrm{~Hz}, 2 \mathrm{H}), 7.26-7.23(\mathrm{~m}, 4 \mathrm{H}), 7.10-7.02(\mathrm{~m}, 3 \mathrm{H}), 6.94$ $(\mathrm{d}, J(\mathrm{H}, \mathrm{H})=8.0 \mathrm{~Hz}, 2 \mathrm{H}), 6.10(\mathrm{~d}, J(\mathrm{H}, \mathrm{H})=8.4 \mathrm{~Hz}, 2 \mathrm{H}), 4.72(\mathrm{t}, J(\mathrm{H}, \mathrm{H})=8.1 \mathrm{~Hz}, 2 \mathrm{H}), 3.74(\mathrm{~s}$, $3 \mathrm{H}), 3.24(\mathrm{t}, J(\mathrm{H}, \mathrm{H})=8.1 \mathrm{~Hz}, 2 \mathrm{H}), 2.60(\mathrm{q}, J(\mathrm{H}, \mathrm{H})=7.6 \mathrm{~Hz}, 2 \mathrm{H}), 1.21(\mathrm{t}, J(\mathrm{H}, \mathrm{H})=7.6 \mathrm{~Hz}$, $3 \mathrm{H}) \mathrm{ppm} .{ }^{13} \mathrm{C} \mathrm{NMR}\left(\mathrm{CD}_{2} \mathrm{Cl}_{2}, \delta\right), 206.6,159.5,143.7,140.0,137.6,131.5,130.7,129.0,128.6$, $126.2,120.2,114.4,112.4,62.4,55.2,31.4,28.3,15.0$ ppm. ${ }^{77} \mathrm{Se} \mathrm{NMR}\left(\mathrm{CDCl}_{3}, \delta\right), 598.4 \mathrm{ppm}$. Accurate mass measurement $\left[\mathrm{CI}^{+}, m / z\right]$ : found $502.0283[\mathrm{M}+\mathrm{H}]^{+}$, calculated mass for $\mathrm{C}_{24} \mathrm{H}_{24}$ BrNOSeH: 502.0285 .

$N$-(4-Chlorophenethyl)-N-(4-ethylphenyl)-4-methoxybenzoselenoamide (11). Reddish yellow paste $(0.21 \mathrm{~g}, 46 \%)$. Selected IR (KBr, cm $\left.{ }^{-1}\right), 1602(\mathrm{~s}), 1508(\mathrm{~s}), 1488(\mathrm{~m}), 1448(\mathrm{~s})$, 1398(s), 1300(m), 1251(vs), 1170(s), 1030(m), 832(m), 809(m). ${ }^{1} \mathrm{H} \mathrm{NMR}\left(\mathrm{CDCl}_{3}, \delta\right), 7.25-7.23$ $(\mathrm{m}, 4 \mathrm{H}), 7.19(\mathrm{~d}, J(\mathrm{H}, \mathrm{H})=8.6 \mathrm{~Hz}, 2 \mathrm{H}), 7.02(\mathrm{~d}, J(\mathrm{H}, \mathrm{H})=8.0 \mathrm{~Hz}, 2 \mathrm{H}), 6.85(\mathrm{~d}, J(\mathrm{H}, \mathrm{H})=8.0 \mathrm{~Hz}$, $2 \mathrm{H}), 6.55(\mathrm{~d}, J(\mathrm{H}, \mathrm{H})=8.0 \mathrm{~Hz}, 2 \mathrm{H}), 6.55(\mathrm{~d}, J(\mathrm{H}, \mathrm{H})=8.0 \mathrm{~Hz}, 2 \mathrm{H}), 4.67(\mathrm{~d}, J(\mathrm{H}, \mathrm{H})=8.0 \mathrm{~Hz}$, $2 \mathrm{H}), 3.69(\mathrm{~s}, 3 \mathrm{H}), 3.21(\mathrm{~d}, J(\mathrm{H}, \mathrm{H})=8.0 \mathrm{~Hz}, 2 \mathrm{H}), 2.54(\mathrm{q}, J(\mathrm{H}, \mathrm{H})=8.1 \mathrm{~Hz}, 2 \mathrm{H}), 1.18(\mathrm{t}$, $J(\mathrm{H}, \mathrm{H})=8.0 \mathrm{~Hz}, 3 \mathrm{H}) \mathrm{ppm} .{ }^{13} \mathrm{C} \mathrm{NMR}\left(\mathrm{CDCl}_{3}, \delta\right), 206.8,159.7,143.7,139.8,136.8,132.5$, $130.4,129.2,128.8,128.7,126.2,112.7,62.8,55.3,31.5,28.4,15.2$ ppm. ${ }^{77}$ Se NMR $\left(\mathrm{CDCl}_{3}, \delta\right)$, 601.5 ppm. Accurate mass measurement $\left[\mathrm{CI}^{+}, m / z\right]$ : found $458.0789[\mathrm{M}+\mathrm{H}]^{+}$, calculated mass for $\mathrm{C}_{24} \mathrm{H}_{24} \mathrm{ClNOSeH}: 458.0790$.

$N$-(4-Bromophenyl)-N-(4-chlorophenethyl)-4-methoxybenzoselenoamide (12). Pale orange paste $(0.20 \mathrm{~g}, 40 \%)$. Selected IR $\left(\mathrm{KBr}, \mathrm{cm}^{-1}\right), 1601(\mathrm{~s}), 1489(\mathrm{~m}), 1485(\mathrm{~s}), 1446(\mathrm{~m})$, 1392(m), 1302(m), 1251(vs), 1170(vs), 1068(m), 1011(m), 832(m), 801(m). ${ }^{1} \mathrm{H} \mathrm{NMR}\left(\mathrm{CDCl}_{3}\right.$,

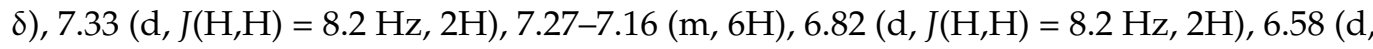
$J(\mathrm{H}, \mathrm{H})=8.3 \mathrm{~Hz}, 2 \mathrm{H}), 4.63(\mathrm{q}, J(\mathrm{H}, \mathrm{H})=8.4 \mathrm{~Hz}, 2 \mathrm{H}), 3.72(\mathrm{~s}, 3 \mathrm{H}), 3.16(\mathrm{~d}, J(\mathrm{H}, \mathrm{H})=8.4 \mathrm{~Hz}$, $3 \mathrm{H}) \mathrm{ppm} .{ }^{13} \mathrm{C} \mathrm{NMR}\left(\mathrm{CDCl}_{3}, \delta\right), 204.8,160.0,145.0,139.6,136.5,132.7,130.3,129.6,129.2$, 128.8, 128.0, 121.1, 113.0, 62.3, 55.4, 29.8 ppm. ${ }^{77} \mathrm{Se} \mathrm{NMR}\left(\mathrm{CDCl}_{3}, \delta\right), 601.4 \mathrm{ppm}$. Accurate mass measurement $\left[\mathrm{CI}^{+}, m / z\right]$ : found $507.9580[\mathrm{M}+\mathrm{H}]^{+}$, calculated mass for $\mathrm{C}_{22} \mathrm{H}_{19}$ BrClNOSeH: 507.9582 . 


\section{Conclusions}

In summary, we have disclosed Woollins' reagent used as a highly efficient chemoselective selenation/reduction reagent for benzamide leading to $\mathrm{N}$-aryl- $\mathrm{N}$-(arylenethyl)benzoselenoamides. The reported results enhance the application of Woollins' reagent further, providing an efficient route to the preparation of the unusual substituted selenoamides.

Author Contributions: G.H. and J.D.W. conceived and designed the project; G.H. performed the experiments; D.B.C., C.L.C.-W. and A.M.Z.S. did the crystallography; All authors have read and agreed to the published version of the manuscript.

Funding: This research received no external funding.

Institutional Review Board Statement: Not applicable.

Informed Consent Statement: Not applicable.

Data Availability Statement: Not applicable.

Acknowledgments: We are grateful to the University of St Andrews for financial support and the EPSRC National Mass Spectrometry Service Centre (Swansea) for mass spectral measurements.

Conflicts of Interest: The authors declare no conflict of interest.

Sample Availability: Samples of all compounds are not available from the authors.

\section{References}

1. Hua, G.; Woollins, J.D. Formation and reactivity of phosphorus-selenium rings. Angew. Chem. Int. Ed. 2009, 48, 1368-1377. [CrossRef] [PubMed]

2. Hua, G.; Zhang, Q.; Li, Y.; Slawin, A.M.Z.; Woollins, J.D. Novel heterocyclic selenazadiphospholaminediselenides, zwitterionic carbamimidoyl(phenyl)phosphinodiselenoic acids and selenoureas derived from cyanamides. Tetrahedron 2009, 65, 6074-6082. [CrossRef]

3. Hua, G.; Li, Y.; Fuller, A.L.; Slawin, A.M.Z.; Woollins, J.D. Facile synthesis and structure of novel 2,5-disubstituted 1,3,4selenadiazoles. Eur. J. Org. Chem. 2009, 2009, 1612-1618. [CrossRef]

4. Gómez Castaño, J.A.; Romano, R.M.; Beckers, H.; Willner, H.; Della Védova, C.O. Trifluoroselenoacetic acid, $\mathrm{CF}_{3} \mathrm{C}(\mathrm{O}) \mathrm{SeH}$ : Preparation and properties. Inorg. Chem. 2010, 49, 9972-9977. [CrossRef]

5. Hua, G.; Li, Y.; Fuller, A.L.; Slawin, A.M.Z.; Woollins, J.D. Synthesis and X-ray structures of new phosphorus-selenium heterocycles with an E-P(Se)-E' (E, E' = N, S, Se) linkage. New J. Chem. 2010, 34, 1565-1571. [CrossRef]

6. Huang, Y.; Jahreis, G.; Lücke, C.; Wildemann, D.; Fischer, G. Modulation of the Peptide Backbone Conformation by the Selenoxo Photoswitch. J. Am. Chem. Soc. 2010, 132, 7578-7579. [CrossRef] [PubMed]

7. Hua, G.; Fuller, A.; Slawin, A.M.; Woollins, J.D. Novel five- to ten-membered organoselenium heterocycles from the selenation of aryl-diols. Eur. J. Org. Chem. 2010, 2010, 2607-2615. [CrossRef]

8. Hua, G.; Henry, J.B.; Li, Y.; Mount, A.R.; Slawin, A.M.Z.; Woollins, J.D. Synthesis of novel 2,5-diarylselenophenes from selenation of 1,4-diarylbutane-1,4-diones or methanol/arylacetylenes. Org. Biomol. Chem. 2010, 8, 1655-1660. [CrossRef]

9. Hua, G.; Fuller, A.L.; Slawin, A.M.; Woollins, J.D. Formation of new organoselenium heterocycles and ring reduction of ten-membered heterocycles into seven-memered heterocycle. Polyhedron 2011, 30, 805-808. [CrossRef]

10. Hua, G.; Fuller, A.L.; Buehl, M.; Slawin, A.M.; Woollins, J.D. Selenation/Thionation of $\alpha$-Amino Acids: Formation and X-ray Structures of Diselenopiperazine and Dithiopiperazine and Related Compounds. Eur. J. Org. Chem. 2011, 2011, $3067-3073$. [CrossRef]

11. Hua, G.; Cordes, D.B.; Li, Y.; Slawin, A.M.; Woollins, J.D. Symmetrical organophosphorus spiroheterocycles from selenation of carbohydrazides. Tetrahedron Lett. 2011, 52, 3311-3314. [CrossRef]

12. Wong, R.C.S.; Ooi, M.L. A new approach to coordination chemistry involving phosphorus-selenium based ligands: Ring opening, deselenation and phosphorus_-phosphorus coupling of Woollins' Reagent. Inorg. Anica Chim. Acta 2011, 366, 350-356. [CrossRef]

13. Gray, I.P.; Bhattacharyya, P.; Slawin, A.M.; Woollins, J.D. A new synthesis of $\left(\mathrm{PhPSe}_{2}\right)_{2}$ (Woollins' Reagnet) and its use in the synthesis of novel P-Se heterocycles. Chem. Eur. J. 2005, 11, 6221-6227. [CrossRef]

14. Hua, G.; Li, Y.; Slawin, A.M.; Woollins, J.D. Synthesis and structure of eight-, nine- and ten-membered rings with P-Se-Se-P linkages. Angew. Chem. 2008, 120, 2899-2901.

15. Hua, G.; Griffin, J.M.; Ashbrook, S.E.; Slawin, A.M.; Woollins, J.D. Octaselenocycododecane. Angew. Chem. Int. Ed. 2011, 123, 4209-4212. [CrossRef]

16. Mandal, M.; Chatterjee, S.; Jaisankar, O. Woollins' reagent: A chemoselective reducing agent for 1,4-enediones and 1,4-ynediones to saturated 1,4-diones. Synlett 2012, 23, 2615. [CrossRef]

17. Pizzo, C.; Graciela, M. Woollins' reagent promotes selective reduction of $\alpha, \beta$-unsaturated thiazo and selenazolidinones. Tetrahedron Lett. 2017, 58, 1445-1447. [CrossRef] 
18. Yu, Y.; Furuyama, T.; Tang, J.; Wu, Z.Y.; Chen, J.Z.; Kobayashi, N.; Zhang, J.L. Stable iso-bacteriochlorin mimics from porpholactone: Effect of $\alpha, \beta$-oxazolone moiety on the frontier $\pi$-molecular orbitals. Inorg. Chem. Front. 2015, 2, 671-677. [CrossRef]

19. Wirth, T. Organoselenium chemistry in stereoselective reactions. Angew. Chem. Int. Ed. 2000, 39, 3740-3749. [CrossRef]

20. Rhoden, C.R.; Zeni, G. New development of synthesis and reactivity of seleno- and tellurophenes. Org. Biomol. Chem. 2011, 9 , 1301-1303. [CrossRef] [PubMed]

21. Uemoto, T.; Emmanuel, M. S- Se-, Te-(perfluoroalkyl)dibenzothiophenium, -selenophenium, and -tellurophenium salts. Adv. Heterocycl. Chem. 1995, 64, 323-339. [CrossRef]

22. Nogueira, C.W.; Zeni, G.; Rocha, J.B.T. Organoselenium and Organotellurium Compounds: Toxicology and Pharmacology. Chemin Rev. 2005, 36, 6255-6286. [CrossRef]

23. Lakner, F.J.; Parker, M.A.; Rogovoy, B.; Khvat, A.; Ivachtchenko, A. Synthesis of novel trisubstituted imidazolines. Synthesis 2009, 12, 1987-1990.

24. Porretta, G.C.; Biava, M.; Fioravanti, R.; Fischetti, M.; Melino, C.; Venza, F.; Bolle, P.; Tita, B. Research on antibacterial and antifungal agents. VIII. Synthesis and antimicrobial activity of 1,4-diarylpyrroles. Eur. J. Med. Chem. 1992, 27, 717-722. [CrossRef]

25. Nishiyama, Y.; Yoshida, M.; Ohkawa, S.; Hamanaka, S. New agents for the selective reduction of the carbon-carbon double bond of alpha/betal unsaturated carbonyl compounds. J. Org. Chem. 1991, 56, 6720-6722. [CrossRef]

26. Mesquita, K.D.; Waskow, B.; Schumacher, R.F.; Perin, G.; Jacob, R.G.; Alves, D. Glycerol/hypophosphorus acid and PhSeSePh: An efficient and selective system for reactions in the carbon-carbon double bond of (E)-chalcones. J. Braz. Chem. Soc. 2014, 25, 1261-1269.

27. Lalezari, I.; Ghanbarpour, F.; Niazi, M.; Jafari-Namin, R. Selenium heterocycles XIV. 2,6-Diaryltetrahydroselenopyran-4-ones. J. Heterocycl. Chem. 1974, 11, 469-470. [CrossRef]

28. Miyashita, M.; Yoshikoehi, A. Facile and highly efficient conjugate addition of benzeneselenol to $\alpha, \beta$-unsaturated carbonyl compounds. Synthesis 1980, 8, 664-666. [CrossRef]

29. Li, Y.; Hua, G.-X.; Slawin, A.M.Z.; Woollins, J.D. The X-ray Crystal Structures of Primary Aryl Substituted Selenoamides. Molecules 2009, 14, 884-892. [CrossRef] [PubMed]

30. CrystalClear-SM Expert v2.1; Rigaku Americas: The Woodlands, TX, USA; Rigaku Corporation: Tokyo, Japan, 2015.

31. CrysAlisPro v1.171.38.43.; Rigaku Oxford Diffraction; Rigaku Corporation: Oxford, UK, 2015.

32. Sheldrick, G.M. SHELXT-Integrated space-group and crystal structure determination. Acta Crystallogr. Sect. A 2015, 71, 3-8. [CrossRef]

33. Beurskens, P.T.; Beurskens, G.; de Gelder, R.; Garcia-Granda, S.; Gould, R.O.; Israel, R.; Smits, J.M.M. DIRDIF-99; Crystallography Laboratory, University of Nijmegen: Nijmegen, The Netherlands, 1999.

34. Sheldrick, G.M. Crystal structure refinement with SHELXL. Acta Crystallogr. Sect. C 2015, 71, 3-8. [CrossRef] [PubMed]

35. CrystalStructure v4.3.0; Rigaku Americas: The Woodlands, TX, USA; Rigaku Corporation: Tokyo, Japan, 2018. 